\title{
Upregulation of T-bet expression in peripheral blood mononuclear cells during Vogt-Koyanagi-Harada disease
}

\author{
B Li, P Yang, H Zhou, X Huang, H Jin, L Chu, Y Gao, L Zhu, A Kijlstra
}

Br J Ophthalmol 2005;89:1410-1412. doi: 10.1136/bjo.2005.074062

Aim: To test the hypothesis that T-bet expression is altered in patients with Vogt-Koyanagi-Harada (VKH) disease.

Methods: Peripheral blood was withdrawn from $16 \mathrm{VKH}$ patients before and after immunosuppressive treatment and from 16 healthy individuals. IFN- $\gamma$, IL-2, and IL- 4 in the serum and the supernatants of peripheral blood mononuclear cells (PBMC) cultured with or without phytohaemagglutinin (PHA) were measured by ELISA. T-bet mRNA and protein expression in PBMC cultured with or without PHA was detected by RT-PCR and western blot, respectively.

Results: The level of IFN- $\gamma$, but not IL-2 and IL-4, was significantly higher in the supernatants of stimulated PBMC in patients than in controls. A significantly increased T-bet mRNA was found in VKH patients during an active uveitis episode, but not in quiescent patients, compared to controls. $\mathrm{T}$-bet protein was detectable in VKH patients during an active uveitis episode, but not in quiescent patients nor in the healthy controls. Stimulation of PBMC with PHA resulted in a marked upregulation of T-bet mRNA and protein expression for both patients and controls with no significant difference between the two groups.

Conclusions: Upregulation of T-bet may be associated with the development of a Th1 mediated immune response in VKH disease.

$\mathrm{V}$ ogt-Koyanagi-Harada (VKH) disease is an autoimmune disorder. ${ }^{1}$ Studies have revealed a strong polarised Thl immune response in VKH patients. ${ }^{2-4}$ T-bet, a transcriptional factor, has been shown to be involved in Thl cell development. It has been shown that T-bet promotes Thl development and IFN- $\gamma$ production, and reprograms a committed population of fully polarised Th2 cells into the Thl phenotype..$^{5-9}$ T-bet also has a key role in controlling Thl cell differentiation and effector functions in vivo. ${ }^{10}{ }^{11}$ T-bet has been implicated in several autoimmune diseases, including Crohn's disease, ${ }^{12}$ coeliac disease, ${ }^{13}$ and Behçet's disease (BD). ${ }^{14}{ }^{15}$ Whether T-bet is also involved in the pathogenesis of VKH disease is not yet known and was therefore the subject of the study reported here. Our results show that Tbet is increased during an active uveitis episode and may thus have a role in the pathogenesis of this disease.

\section{PATIENTS AND METHODS Patients}

Sixteen VKH patients ( 10 men and six women) with an average age of 37 years and 16 healthy individuals ( 10 men and six women) with an average age of 35 years were included in this study. The diagnosis of VKH disease was based on the revised criteria. ${ }^{16}$ All patients showed recurrent intraocular inflammation and had been intermittently treated with prednisone. Patients were referred to our centre with uveitis, showing mutton fat keratic precipitates (KP), cells in the anterior chamber, Dalen-Fuchs nodules, and sunset glow fundus. The extraocular findings included vitiligo $(25 \%)$, alopecia $(43.8 \%)$, dysacusis $(25 \%)$, tinnitus $(56.3 \%)$, and poliosis (43.8\%). Uveitis was completely controlled in all the patients after treatment with chlorambucil combined with prednisone for at least 1 year. Blood samples were collected before this treatment (active uveitis stage) and 3 months after stopping the treatment (inactive uveitis stage). The blood samples were concurrently obtained from the matched healthy individuals. All procedures followed the tenets of the Declaration of Helsinki, and informed consent was obtained from all patients and healthy controls.

\section{Preparation of samples}

The blood samples were used for isolation of serum and peripheral blood mononuclear cells (PBMC). PBMC from each patient were divided into two aliquots: one directly for analysis of T-bet, and the other for analysis of T-bet following stimulation with phytohaemagglutinin (PHA). For the latter, the PBMC were cultured in RPMI 1640 with PHA (Sigma, MO, USA) at a concentration of $5 \mu \mathrm{g} / \mathrm{ml}$ for 72 hours and subsequently used for T-bet analysis by RT-PCR and western blot. The culture supernatants were stored at $-80^{\circ} \mathrm{C}$ for cytokine assays.

\section{Cytokine assays}

Cytokine levels of IFN- $\gamma$, IL-2, and IL- 4 in the serum and supernatants of PHA stimulated PBMC were assayed by ELISA. Procedures were preformed according to the manufacturer's instructions ( $\mathrm{R} \& \mathrm{D}$ systems, MN, USA). The sensitivity of IFN- $\gamma$, IL-2, and IL-4 were $8 \mathrm{pg} / \mathrm{ml}, 7 \mathrm{pg} / \mathrm{ml}$, and $10 \mathrm{pg} / \mathrm{ml}$, respectively.

\section{RT-PCR and western blot analysis}

RT-PCR and western blot analysis were performed using the reagents and procedures described previously. ${ }^{14}$

\section{Statistical analysis}

Data were reported as mean (SD). The paired $t$ test was used for statistical analysis. A level of $\mathrm{p}<0.05$ was accepted as statistically significant.

\section{RESULTS}

Cytokine concentrations in the serum and supernatants of cultured PBMC

IFN- $\gamma$, IL-2, or IL-4 was undetectable in the serum from either VKH patients or controls. IFN- $\gamma$ concentrations in the supernatants of stimulated PBMC during active and inactive uveitis episodes were 645.47 (32.3) pg/ml and 565.61 (74.06)

Abbreviations: $B D$, Behçet's disease; $K P$, keratic precipitates; PBMC, peripheral blood mononuclear cells; PHA, phytohaemagglutinin; VKH, Vogt-Koyanagi-Harada 


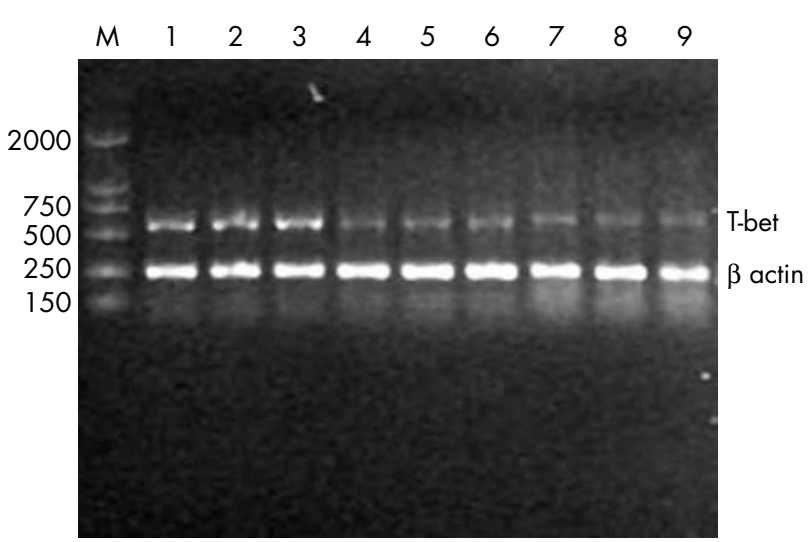

Figure 1 RT-PCR products of T-bet mRNA extracted from freshly obtained PBMC of three patients before (lanes 1, 2,3) and after (lanes 4 , $5,6)$ treatment and from three controls (lanes $7,8,9)$. One representative experiment is presented out of the 16 patients with or without active uveitis and 16 controls.

$\mathrm{pg} / \mathrm{ml}$, respectively, and showed no significant difference $(p>0.05)$. They were both significantly higher than concentrations in controls $(28.94(6.76) \mathrm{pg} / \mathrm{ml})(\mathrm{p}<0.001)$. IL-2 and IL-4 contents were both below the detection level in the supernatants of PHA stimulated PBMC from the patients and controls.

\section{Expression of T-bet mRNA in patients and controls}

The sequenced polymerase chain reaction (PCR) products showed a $99.6 \%$ homology with the known T-bet sequence. T-bet mRNA transcripts were observed in all the tested samples (fig 1). T-bet mRNA band intensity was normalised by the respective $\beta$ actin mRNA band and was found to be 0.74 in patients with active uveitis. This T-bet mRNA level was significantly higher than that in patients with inactive uveitis (0.18) as well as in controls $(0.17) \quad(\mathrm{p}<0.001)$. However, there was no significant correlation between T-bet mRNA expression and the severity of the intraocular inflammation in patients with active uveitis. No difference

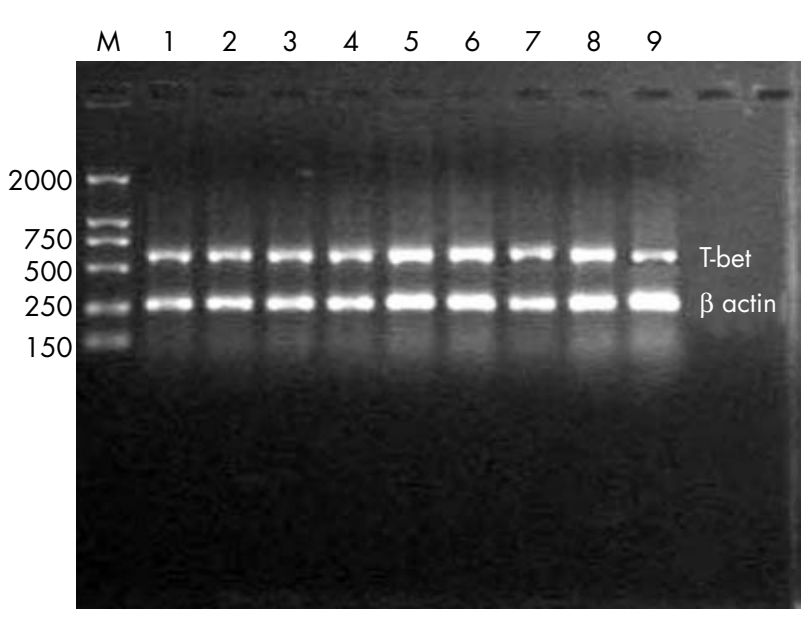

Figure 2 RT-PCR products of T-bet mRNA extracted from PHA stimulated PBMC from three patients with active (lanes 1, 2, 3) and inactive (lanes $4,5,6)$ uveitis and three controls (lanes $7,8,9)$. One representative experiment is presented out of the 16 patients with or without active uveitis and 16 controls.

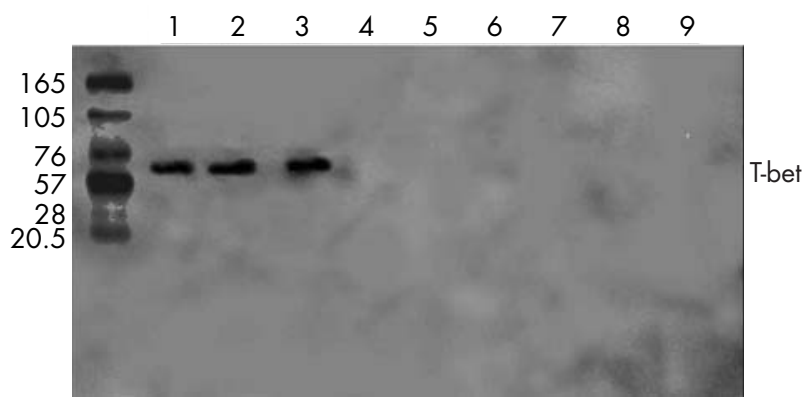

Figure 3 Western blot analysis of T-bet protein from freshly obtained PBMC of patients and controls. A $62 \mathrm{kDa}$ protein is detectable in samples from patients with active uveitis (lanes 1, 2, 3), but not in patients with inactive uveitis (lanes 4, 5, 6) and controls (lanes 7, 8, 9). One representative experiment of five independent experiments is shown.

was found between quiescent patients and controls concerning this ratio. Moreover, there was no difference in this ratio between VKH patients with (0.81) or without (0.84) active uveitis and controls (0.82) after PHA stimulation (fig 2).

\section{Expression of T-bet protein in patients and controls}

T-bet protein was assayed using western blot. Before PHA treatment, a protein band of approximately $62 \mathrm{kDa}$ could only be detected in the PBMC from patients with active uveitis, but not in those from patients with inactive uveitis or the controls (fig 3). In contrast, after PHA stimulation, all the tested samples showed the $62 \mathrm{kDa}$ band. Furthermore, quantitative analysis indicated that the expression of T-bet protein after stimulation was essentially identical in patients and controls (fig 4).

\section{DISCUSSION}

Our study revealed an upregulated T-bet expression at both mRNA and protein levels, in association with a significantly increased IFN- $\gamma$ in the supernatants of PHA stimulated PBMC, in VKH patients with active uveitis. These results suggest that $\mathrm{T}$ cells in PBMC in VKH disease are apt to deviate towards the Thl type and that T-bet may have a role in the pathogenesis of VKH disease.

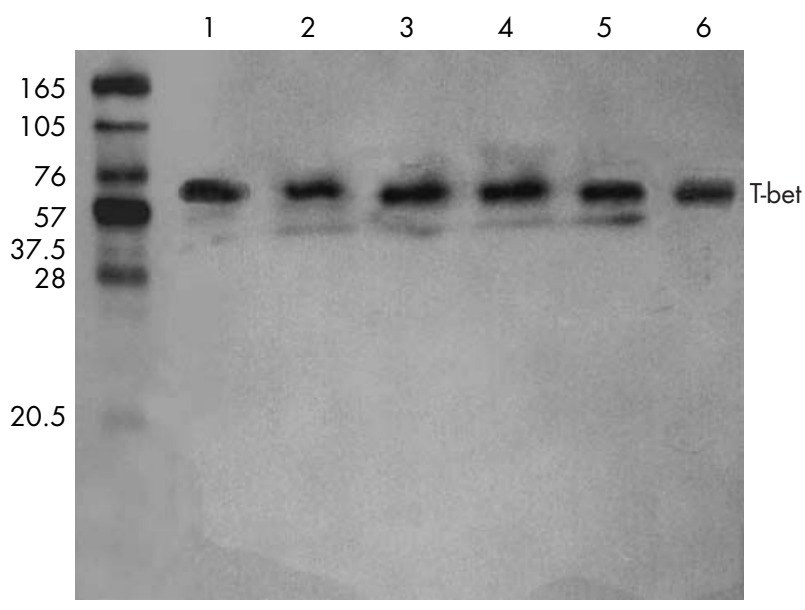

Figure 4 T-bet protein expression in PHA-stimulated PBMC from patients and controls. A $62 \mathrm{kDa}$ protein was detectable in all tested samples from patients with active (lanes 1,2) or inactive uveitis (lanes 3 , 4) and controls (lanes 5, 6). One representative experiment of eight independent experiments is shown. 
T-bet expression has been shown to be increased in activated $\mathrm{T}$ cells and is the key transcriptional factor for Thl cell activation and cytokine production. ${ }^{68}$ Our findings are in agreement with studies on T-bet expression in other autoimmune diseases, such as Crohn's disease, ${ }^{12}$ coeliac disease, ${ }^{13}$ and Behçet's disease (BD). ${ }^{14}$ The observation that $\mathrm{T}$-bet expression is increases in $\mathrm{VKH}$ patients during an active uveitis episode, but not during inactive uveitis supports a role of activated $\mathrm{T}$ cells during the pathogenesis of this disease. In addition, our study also showed that a significantly upregulated T-bet expression was readily detected in the quiescent patients and controls after PHA stimulation. However, no marked difference was found in the expression of T-bet in patients with active uveitis before and after stimulation. These results may suggest that a large number of T-bet ${ }^{+}$cells already exist in the patients with active uveitis, and only few T-bet ${ }^{+}$cells are present in patients with inactive uveitis. Quiescent patients in that respect are similar to the controls. Therefore, T-bet may be closely associated with activity of VKH disease and may serve as an immunomodulatory target.

The increased T-bet expression in VKH patients with active uveitis in this study is consistent with the results in BD patients with active uveitis. ${ }^{14}$ It is interesting to note that there are marked differences in clinical and pathological features between these two uveitis entities. The similar results in T-bet expression observed in VKH patients and BD patients, however, suggest a common pathway involved in their pathogenesis.

Given the fact that T-bet can be expressed in both $\mathrm{CD}^{+} \mathrm{T}$ cells and B cells, future studies should address the exact cellular origin of T-bet ${ }^{+}$cells using multicolour FACS colocalisation studies. The fact that T-bet upregulation is associated with a concomitant IFN- $\gamma$ expression points to the $\mathrm{T}$ cell as the most likely source. Whether T-bet is an exclusive marker of cell activation during VKH is not known and studies investigating on the correlation between T-bet and other $\mathrm{CD}^{+}$cell activation markers such as CD25 and CD69 may shed more light on this issue. Additionally, further evidence implicating T-bet in the pathogenesis of uveitis will involve the use of T-bet blocking reagents or T-bet knockout mice, which may open a new venue for the treatment of human uveitis.

\section{ACKNOWLEDGEMENT}

This work was supported by the Fund for Innovative Research Groups (30321004), National Nature Science Foundation (30400487).

\section{Authors' affiliations}

B Li, P Yang, H Zhou, X Huang, H Jin, L Chu, Y Gao, L Zhu, Zhongshan Ophthalmic Center, Sun Yat-sen University, Guangzhou, P R China B Li, P Yang, H Zhou, X Huang, Y Gao, L Zhu, Uveitis Study Center, Sun Yat-sen University, Guangzhou, P R China

H Jin, Division of Immunology, Children's Hospital Boston, Harvard Medical School, Boston, MA 02115, USA

A Kijlstra, Animal Sciences Group, Wageningen University and Research Center, Lelystad, Eye Research Institute Maastricht, and Department of Ophthalmology, Academic Medical Center, University of Amsterdam, Netherlands

Correspondence to: Professor Peizeng Yang, Zhongshan Ophthalmic Center, Sun Yat-sen University, 54 Xianlie Road, Guangzhou 510060, P $\mathrm{R}$ China; peizengyang@hotmail.com

Accepted for publication 15 June 2005

\section{REFERENCES}

1 Rao NA. Mechanisms of inflammation response in symphathetic ophthalmia and Vogt-Koyanagi-Harada syndrome. Eye 1997;11(pt 2):213-16.

2 Norose K, Yano A, Wang XC, et al. Dominance of activated T cells and IL-6 in aqueous humor in Vogt-Koyanagi Harada disease. Invest Ophthalmol Vis Sci 1994;35:33-9.

3 Imai $Y$, Sugita M, Nakamura S, et al. Cytokine production and helper T cell subsets in Vogt-Koyanagi-Harada disease. Curr Eye Res 2001;22:312-18.

4 Sakaguchi M, Sugita S, Sugawa K, et al. Cytokine production by T cells infiltrating in the eye of uveitis patients. Jpn J Ophthalmol 1998;42:262-8.

5 Szabo SJ, Sullivan BM, Peng SL, et al. Molecular mechanisms regulating TH1 immune response. Annu Rev Immunol 2003;21:713-58.

6 Szabo SJ, Kim ST, Costa GL, et al. A novel transcription factor, T-bet, direct Th1 linage commitment. Cell 2000;100:655-69.

7 Papaioannou VE. T-box genes in development: from hydra to humans. Int Rev Cytol 2001;207:1-70.

8 Mullen AC, High FA, Hutchins AS. Role of T-bet in commitment of TH1 cells before IL-12 development selection. Science 2001;292:1907-10.

9 Agnello D, Lankford CS, Bream J, et al. Cytokines and transcription factors that regulate T helper cell differentiation: new players and new insights. J Clin Immunol 2003;23:147-61.

10 Hultgren $\mathrm{OH}$, Verdrengh $M$, Tarkowski A. T-box transcription-factor-deficient mice display increased joint pathology and failure of infection control during staphylococcal arthritis. Microbes Infect 2004;6:529-35.

11 Szabo SJ, Sullivan BM, Stemmann C. Distinct effects of T-bet in TH1 lineage commitment and IFN-gamma production in CD4 and CD8 T cells. Science 2002;295:338-42.

12 Matsuoka K, Inoue N, Sato T, et al. T-bet upregulation and subsequent interleukin 12 stimulation are essential for induction of Th 1 mediated immunopathology in Crohn's disease. Gut 2004:53:1303-8.

13 Holtmann MH, Neurath MF. T helper cell polarisation in coeliac disease: any (T-)bet? Gut 2004;53:1065-7.

14 Li B, Yang P, Zhou H, Zhang Z, et al. T-bet expression is upregulated in active Behçet's disease. Br J Ophthalmol 2003;87:1264-7.

15 Rajendram R, Rao NA. Molecular mechanisms in Behçet's disease. Br J Ophthalmol 2003;87:1 199-200.

16 Read RW, Holland GN, Rao NA, et al. Revised diagnostic criteria for VogtKoyanagi-Harada disease: report of an international committee on nomenclature. Am J Ophthalmol 2001;131:647-52. 